\title{
Neutrino factory
}

M. Bogomilov, ${ }^{1}$ R. Matev, ${ }^{1}$ R. Tsenov, ${ }^{1}$ M. Dracos, ${ }^{2}$ M. Bonesini, ${ }^{3}$ V. Palladino, ${ }^{4}$ L. Tortora, ${ }^{5}$ Y. Mori ${ }^{6}{ }^{6}$ T. Planche,,${ }^{6,}$ J. B. Lagrange, ${ }^{6, \dagger}$ Y. Kuno, ${ }^{7}$ E. Benedetto, ${ }^{8}$ I. Efthymiopoulos, ${ }^{8}$ R. Garoby, ${ }^{8}$ S. Gilardoini, ${ }^{8}$ M. Martini, ${ }^{8}$ E. Wildner, ${ }^{8}$ G. Prior, ${ }^{8,}$ A. Blondel,${ }^{9}$ Y. Karadzhow, ${ }^{9}$ M. Ellis, ${ }^{10,8}$ P. Kyberd, ${ }^{10}$ R. Bayes,${ }^{11}$ A. Laing, ${ }^{11}$ F. J. P. Soler ${ }^{11}$ A. Alekou, ${ }^{12}$ M. Apollonio, ${ }^{12, \|}$ M. Aslaninejad, ${ }^{12}$ C. Bontoiu, ${ }^{12}$ L. J. Jenner, ${ }^{12}$ A. Kurup, ${ }^{12}$ K. Long, ${ }^{12}$ J. Pasternak, ${ }^{12}$ A. Zarrebini, ${ }^{12}$ J. Poslimski, ${ }^{12, \mid}$ V. Blackmore, ${ }^{13}$ J. Cobb,${ }^{13}$ C. Tunnell, ${ }^{13}$ C. Andreopoulos,${ }^{14}$ J. R. J. Bennett, ${ }^{14}$ S. Brooks, ${ }^{14}$ O. Caretta,${ }^{14}$ T. Davenne, ${ }^{14}$ C. Densham,${ }^{14}$ T. R. Edgecock,${ }^{14}$ M. Fitton, ${ }^{14}$ D. Kelliher, ${ }^{14}$ P. Loveridge,${ }^{14}$ A. McFarland,${ }^{14}$ S. Machida, ${ }^{14}$ C. Prior, ${ }^{14}$ G. Rees,${ }^{14}$ C. Rogers ${ }^{14}$ M. Rooney, ${ }^{14}$ J. Thomason, ${ }^{14}$ D. Wilcox,${ }^{14}$ C. Booth,${ }^{15}$ G. Skoro, ${ }^{15}$ J. J. Back ${ }^{16}$ P. Harrison, ${ }^{16}$ J. S. Berg, ${ }^{17}$ R. Fernow, ${ }^{17}$ J. C. Gallardo, ${ }^{17}$ R. Gupta, ${ }^{17}$ H. Kirk ${ }^{17}$ N. Simos, ${ }^{17}$ D. Stratakis, ${ }^{17}$ N. Souchlas, ${ }^{17}$ H. Witte, ${ }^{17}$ A. Bross,${ }^{18}$ S. Geer, ${ }^{18}$ C. Johnstone, ${ }^{18}$ N. Makhov,${ }^{18}$ D. Neuffer, ${ }^{18}$ M. Popovic, ${ }^{18}$ J. Strait, ${ }^{18}$ S. Striganov, ${ }^{18}$ J. G. Morfín, ${ }^{18}$ R. Wands, ${ }^{18}$ P. Snopok, ${ }^{19}$ S. A. Bagacz, ${ }^{20}$ V. Morozov, ${ }^{20}$ Y. Roblin, ${ }^{20}$ D. Cline,${ }^{21}$ X. Ding,${ }^{21}$ C. Bromberg, ${ }^{22}$ T. Hart,${ }^{24}$ R. J. Abrams, ${ }^{24}$ C. M. Ankenbrandt,${ }^{24}$ K. B. Beard, ${ }^{24}$ M. A. C. Cummings, ${ }^{24}$ G. Flanagan, ${ }^{24}$ R. P. Johnson, ${ }^{24}$ T. J. Roberts, ${ }^{24}$ C. Y. Yoshikawa, ${ }^{24}$ V. B. Graves ${ }^{25}$ K. T. McDonald, ${ }^{26}$ L. Coney, ${ }^{27}$ and G. Hanson ${ }^{27}$

${ }^{1}$ Department of Atomic Physics, St. Kliment Ohridski University of Sofia, 5 James Bourchier Boulevard, BG-1164 Sofia, Bulgaria

${ }^{2} I P H C$, Université de Strasbourg, CNRS/IN2P3, F-67037 Strasbourg, France ${ }^{3}$ INFN, Sezione Milano Bicocca, Piazza Scienza 3, Milano, Italy

${ }^{4}$ Dipartimento di Scienze Fisiche dell'Universitá di Napoli and INFN, 80126 Napoli, Italy ${ }^{5}$ INFN, Sezione Roma 3, Roma, Italy

${ }^{6}$ Kyoto University, Research Reactor Institute, 2,Asashiro-Nishi, Kumatori-cho, Sennan-gun, Osaka 590-0494 Japan

${ }^{7}$ Osaka University, Graduate School, School of Science, 1-1 Machikaneyama-cho, Toyonaka, Osaka 560-0043, Japan

${ }^{8}$ CERN, CH-1211 Geneva 23, Switzerland

${ }^{9}$ University de Geneve, 24, Quai Ernest-Ansermet, 1211 Geneva 4, Suisse

${ }^{10}$ Brunel University West London, Uxbridge, Middlesex UB8 3PH, United Kingdom

${ }^{11}$ School of Physics and Astronomy, University of Glasgow, Glasgow G12 8QQ, United Kingdom

${ }^{12}$ Physics Department, Blackett Laboratory, Imperial College London, Exhibition Road, London SW7 2AZ, United Kingdom

${ }^{13}$ Particle Physics Department, The Denys Wilkinson Building, Keble Road, Oxford OX1 3RH, United Kingdom

${ }^{14}$ STFC Rutherford Appleton Laboratory, Didcot, Oxon OX11 OQX, United Kingdom

${ }^{15}$ University of Sheffield, Dept. of Physics and Astronomy, Hicks Bldg., Sheffield S3 7RH, United Kingdom

${ }^{16}$ University of Warwick, Coventry CV4 7AL, United Kingdom

${ }^{17}$ Brookhaven National Lab, P.O. Box 5000, Upton, New York 11973-5000, USA

${ }^{18}$ Fermi National Accelerator Laboratory, Batavia, Illinois 60510-5011, USA

${ }^{19}$ Illinois Institute of Technology, $3101 \mathrm{~S}$ Dearborn St, Chicago, Illinois 60616, USA

${ }^{20}$ Thomas Jefferson National Accelerator Facility, Newport News, Virginia 23606, USA

${ }^{21}$ Department of Physics and Astronomy, University of California, Los Angeles, California 90095, USA

${ }^{22}$ Michigan State University, 150 Administration Building, East Lansing, Michigan 48824, USA

${ }^{23}$ The University of Mississippi, Department of Physics and Astronomy, 108 Lewis Hall, PO Box 1848, Oxford, Mississippi 38677-1848, USA

\footnotetext{
*Now at TRIUMF, 4004 Wesbrook Mall, Vancouver, B.C., V6T 2A3, Canada.

"Now at Imperial College London, Exhibition Road, London SW7 2AZ, UK.

*Now at Laboratório de Instrumentacão e Física Experimental de Particulas, Lisboa, Portugal.

${ }^{\S}$ Now at Westpac Institutional Bank, Sydney, NSW, Australia.

${ }^{\|}$Now at DLS, Harwell Science and Innovation Campus, Didcot, Oxon, OX11 ODEM, UK.

${ }^{\text {I }}$ Corresponding author. j.pozimski@imperial.ac.uk
} 


\author{
${ }^{24}$ Muons Inc., 552N. Batavia Avenue, Batavia, Illinois 60510, USA \\ ${ }^{25}$ Oak Ridge National Laboratory, P.O. Box 2008, Oak Ridge, Tennessee 37831, USA \\ ${ }^{26}$ Princeton University, Princeton, New Jersey 08544, USA \\ ${ }^{27}$ Department of Physics and Astronomy, University of California, Riverside, California 92521, USA
}

(Received 10 August 2014; published 8 December 2014) standard model. The study of subleading effects in neutrino oscillations, and the race to discover $\mathrm{CP}$ invariance violation in the lepton sector, has begun with the recent discovery that $\theta_{13}>0$. The measured value of $\theta_{13}$ is large, emphasizing the need for a facility at which the systematic uncertainties can be reduced to the percent level. The neutrino factory, in which intense neutrino beams are produced from the decay of muons, has been shown to outperform all realistic alternatives and to be capable of making measurements of the requisite precision. Its unique discovery potential arises from the fact that only at the neutrino factory is it practical to produce high-energy electron (anti)neutrino beams of the required intensity. This paper presents the conceptual design of the neutrino factory accelerator facility developed by the European Commission Framework Programme 7 EURO $\nu$ Design Study consortium. EURO $\nu$ coordinated the European contributions to the International Design Study for the Neutrino Factory (the IDS-NF) collaboration. The EURO $\nu$ baseline accelerator facility will provide $10^{21}$ muon decays per year from $12.6 \mathrm{GeV}$ stored muon beams serving a single neutrino detector situated at a source-detector distance of between $1500 \mathrm{~km}$ and $2500 \mathrm{~km}$. A suite of near detectors will allow definitive neutrino-scattering experiments to be performed.

DOI: 10.1103/PhysRevSTAB.17.121002

PACS numbers: $14.60 . P q$

\section{INTRODUCTION}

The phenomenon of neutrino oscillations has been established through measurements of neutrinos produced in the sun, neutrinos produced by cosmic-ray interactions in the atmosphere, antineutrinos produced in nuclear reactors, and using neutrino beams produced by high-energy particle accelerators [1]. The bulk of the data has been collected using the dominant, "disappearance" channels $\stackrel{(-)}{\nu}_{e} \rightarrow \stackrel{(-)}{\nu}_{e}$ and $\stackrel{(-)}{\nu}_{\mu} \rightarrow \stackrel{(-)}{\nu}_{\mu}$. The Daya Bay [2,3], RENO [4] and Double Chooz $[5,6]$ experiments have presented evidence for subleading oscillations through the disappearance of $\bar{\nu}_{e}$. T2K has recently observed $\nu_{e}$ appearance in a $\nu_{\mu}$ beam [7-9]. These discoveries are exciting since they indicate that it is conceivable that $C P$-invariance violation in the lepton sector can be discovered in long-baseline neutrinooscillation experiments.

Neutrino oscillations arise from the "beating" of the phase of neutrino-mass eigenstates as a neutrino produced in an eigenstate of flavor travels through space and time. The three flavor eigenstates, $\nu_{e}, \nu_{\mu}$, and $\nu_{\tau}$ may be written as linear superpositions of the mass eigenstates, $\nu_{1}, \nu_{2}$, and $\nu_{3}$. To describe neutrino oscillations, the matrix by which the neutrino-mass basis is rotated into the neutrino-flavor basis is usually parametrized in terms of three mixing angles

Published by the American Physical Society under the terms of the Creative Commons Attribution 3.0 License. Further distribution of this work must maintain attribution to the author $(s)$ and the published article's title, journal citation, and DOI. $\left(\theta_{12}, \theta_{23}\right.$, and $\left.\theta_{13}\right)$ and one phase parameter $(\delta)$ [10-13]. If $\delta$ is nonzero, $C P$-invariance violation will occur in neutrino oscillations via the neutrino-mixing matrix. Measurements of neutrino oscillations are not sensitive to the neutrino masses themselves, but may be used to determine the mass-squared differences $\Delta m_{31}^{2}=m_{3}^{2}-m_{1}^{2}$ and $\Delta m_{21}^{2}=m_{2}^{2}-m_{1}^{2}$. The sign of $\Delta m_{31}^{2}$ determines the neutrino mass hierarchy; the "normal hierarchy" $(\mathrm{NH})$ refers to the case in which the mass eigenstate $\nu_{3}$ is heavier than the other two neutrinos while the case in which $\nu_{3}$ is lighter than the other two is referred to as the "inverted hierarchy" (IH). By fitting the world's oscillation data, it has been possible to determine $\theta_{12}$ at the $\sim 2.5 \%$ level and $\theta_{13}$ at the $\sim 5 \%$ level [14]. The fit is able to determine that $\left|\theta_{23}-45^{\circ}\right| \sim 5^{\circ}$ with a precision of approximately $3 \%-5 \%$, but is unable to determine the sign of $\left(\theta_{23}-45^{\circ}\right)$. The same fit yields a value for $\Delta m_{21}^{2}\left(\sim 7.5 \times 10^{-5} \mathrm{eV}^{2}\right)$ and a value for $\left|\Delta m_{31}^{2}\right|\left(\sim 2.5 \times 10^{-3} \mathrm{eV}^{2}\right)$. The sign of $\Delta m_{31}^{2}$ is unknown and the $C P$-violating phase, $\delta$, is at present essentially unconstrained.

The challenge to the neutrino community is to measure all the mixing angles as precisely as possible, to determine the sign of $\left(\theta_{23}-45^{\circ}\right)$ and the sign of $\Delta m_{31}^{2}$, to measure $\Delta m_{21}^{2}$ and $\Delta m_{31}^{2}$ precisely, and, by measuring $\delta$, to discover leptonic $C P$-violation if it occurs. Precise measurements of the parameters that govern neutrino oscillations are essential if a complete understanding of the nature of the neutrino is to be obtained. The ultimate theory must surely unify the quark and lepton sectors; so, for the experimentalist, the goal must be to measure the neutrino-mixing parameters with an uncertainty that matches the precision with which the quark-mixing parameters are known. Such 

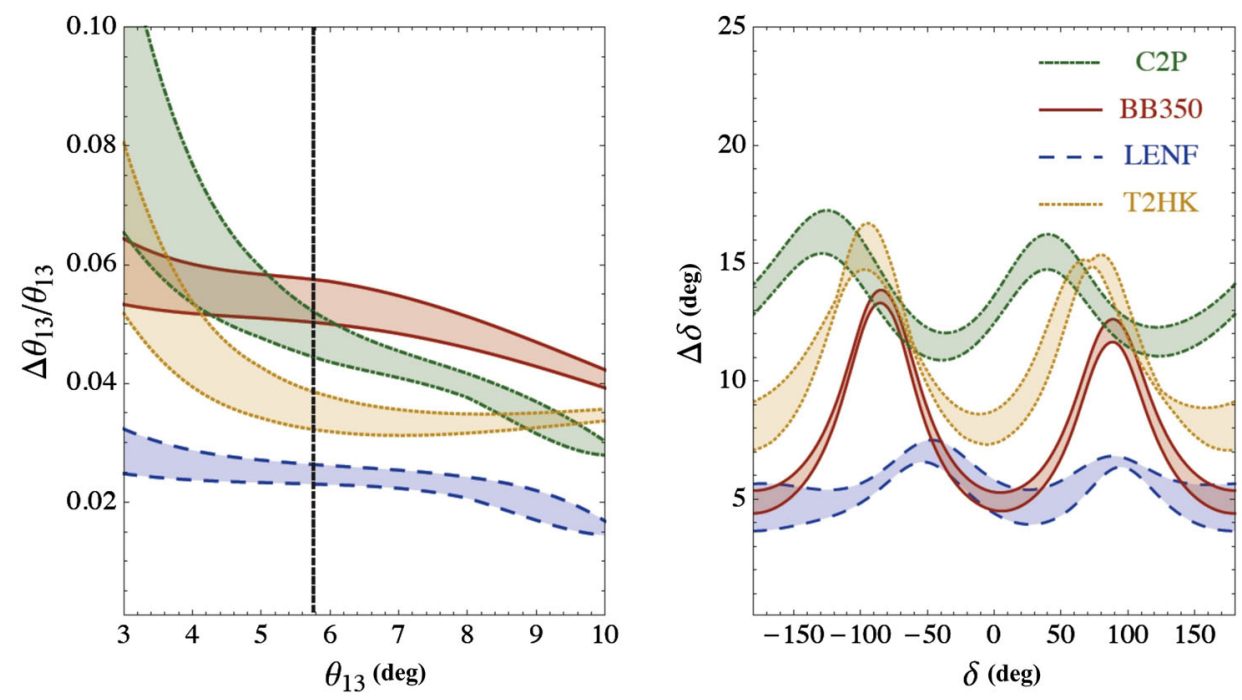

FIG. 1. Precision on $\theta_{13}$ (left panel) and $\delta$ (right panel) [16]. The EURO $\nu$ baseline neutrino factory (labeled LENF) is shown by the blue dashed lines. The normal hierarchy has been assumed. For comparison, the performance of the CERN to Pyhäsalmi super-beam experiment (labeled C2P, green, dashed-dotted lines) [17], T2HK (yellow, dotted lines) [18] and the $\gamma=350$ beta-beam (red, solid lines) [19] are shown. The width of the bands in each panel represent the dependence of the uncertainty of $\theta_{13}$ on $\delta$ (left panel) and the dependence of the uncertainty on $\delta$ on $\theta_{13}$ when it is varied in the range $5.7-10^{\circ}$ (right panel).

measurements will either establish the minimal model outlined above or, by establishing parameter sets inconsistent with it, point to the existence of entirely new phenomena.

In the neutrino factory, beams of (anti)electron and (anti) muon-neutrinos are produced from the decay of muons circulating in a storage ring [15]. As the ratio of the mass of the muon to that of the electron is large, the neutrinos carry away a substantial fraction of the energy of the parent muon, hence, high neutrino energies can readily be achieved. Charged-current interactions induced by "golden channel," $\nu_{e} \rightarrow \nu_{\mu}$ oscillations produce muons of charge opposite to those produced by the antimuon neutrinos in the beam and thus a magnetized detector is required. The additional capability to investigate the "silver" $\left(\nu_{e} \rightarrow \nu_{\tau}\right)$ channel and perhaps also the "platinum" $\left(\nu_{\mu} \rightarrow \nu_{e}\right)$ channel makes the neutrino factory the ideal place to look for oscillation phenomena that are outside the standard, three-neutrino-mixing paradigm. It is thus the ideal facility to serve the precision era of neutrino oscillation measurements.

The performance of the baseline neutrino factory described below is compared to the beta-beam and super-beam alternatives studied by the $\mathrm{EURO} \nu$ consortium in Fig. 1 [16]. With $10^{21}$ muon decays per year at a stored muon energy of $12.6 \mathrm{GeV}$ serving a $100 \mathrm{kT}$ Magnetized Iron Neutrino Detector (MIND) sited at a distance of $2000 \mathrm{~km}$ from the source, the neutrino factory significantly outperforms the other options. Figure 1 shows the excellent precision with which $\delta$ can be measured at the neutrino factory [16].
The performance of the neutrino factory has been considered in detail in $[16,20,21]$. In addition to the excellent precision and outstanding discovery reach, the neutrino factory has great sensitivity to nonstandard neutrino interactions, in particular to those that result in an anomalous rate of $\tau^{ \pm}$production. These properties make the neutrino factory the facility of choice to serve the precision era of neutrino oscillation measurements.

\section{ACCELERATOR FACILITY}

\section{A. Overview}

A schematic diagram of the neutrino factory accelerator facility is shown in Fig. 2. Pions are produced by the interaction of high-energy protons with a target. The pions are captured in a large-aperture, high-field solenoid and transported to a solenoid-focusing decay channel. In the decay channel, muons of both signs are produced, captured and focused in a channel designed to maximize the number of muons transported to the muon storage ring. The captured muons have a large energy spread and a large transverse emittance, both of which need to be reduced, so that the beam can be accelerated efficiently. In the muon front-end, the bunching and phase-rotation sections reduce the energy spread. The transverse emittance is then reduced by a factor of $\sim 2$ using ionization cooling. The MICE experiment which is under construction at RAL [22] will prove the principle of ionization cooling and allow for precise analyses of the cooling performance. A sequence of accelerator systems is used to accelerate the beam to its final energy of $12.6 \mathrm{GeV}$. The first stage of acceleration is performed using a linac, because the large transverse 


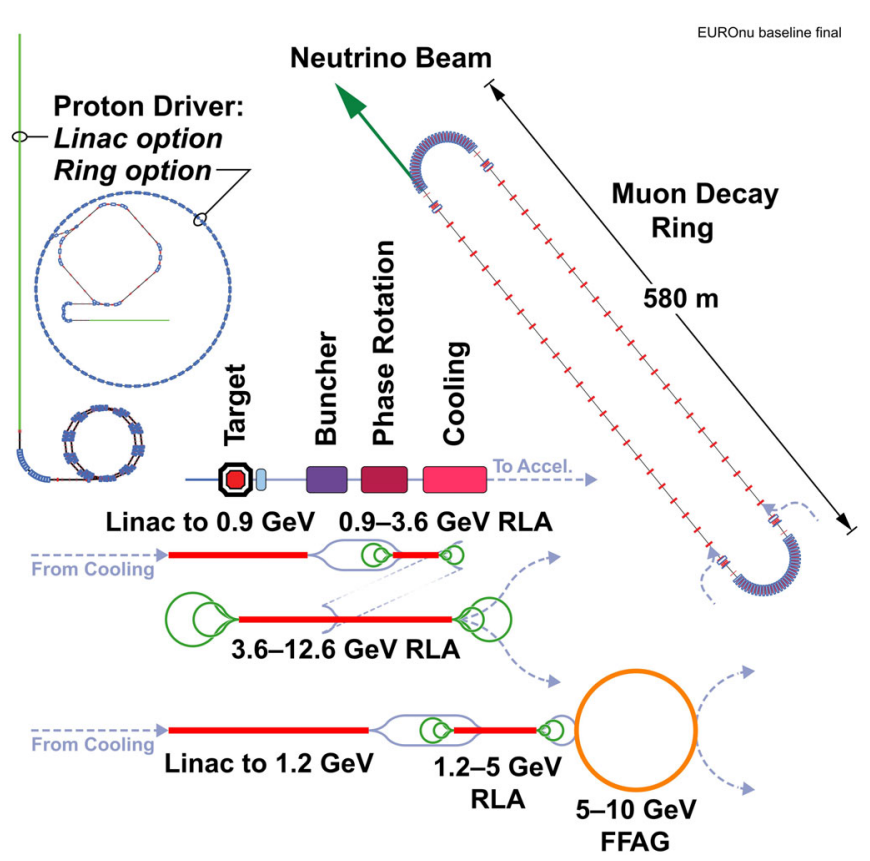

FIG. 2. Schematic diagram of the Neutrino Factory accelerator facility. The function of the various accelerator systems is described in the text. The design is a development of that described in [21].

emittance and energy spread, combined with the variation of velocity with energy, make it impractical to recirculate the low-energy beam. The linac is followed by one or two recirculating linear accelerators (RLAs) in each of which the beam makes multiple passes through the accelerating structures. In the case of the one-RLA scenario, the final stage of acceleration is performed using a linear nonscaling fixed field alternating gradient (FFAG) accelerator which allows many more passes through the cavities. Finally, the beam is injected into one racetrack-shaped decay ring, the straight sections of which are pointed at a single far detector at a distance of between $1500 \mathrm{~km}$ and $2500 \mathrm{~km}$.

\section{History}

In 2005 a one-year review, the International Scoping Study for a future neutrino factory and super-beam facility (the ISS) [23], assessed the status of neutrino factory work and identified a self-consistent and viable conceptual design for the accelerator facility. In 2007, for the case of a large $\sin ^{2} 2 \theta_{13}$, options for a low energy neutrino factory have been published in [24]. The ISS scheme was subsequently adopted by its successor, the International Design Study for the neutrino factory (the IDS-NF) collaboration [25], which delivered an Interim Design Report (IDR) [21] in 2011 and is due to deliver a Reference Design Report (RDR) in 2014. The IDR presented two options for the facility, one optimized for sensitivity to $\delta$ for values of $\sin ^{2} 2 \theta_{13}$ as low as $10^{-4}$ and a second optimized for large $\sin ^{2} 2 \theta_{13} \sim 0.1$. The RDR will include a specification for the accelerator complex, initial consideration of engineering constraints, identification of the R\&D needs and an estimate of the cost of the facility.

The four-year program of the EURO $\nu$ consortium started in 2008. For the neutrino factory, EURO $\nu$ coordinated the European contributions to the IDS-NF. The measurement of $\theta_{13}$ presented by the Daya Bay collaboration early in 2012 caused the IDS-NF collaboration to adopt the large $\theta_{13}$ option presented in its IDR as the baseline for the facility. For EURO $\nu$, the Daya Bay result came just a few months before the study was due to report. Preliminary studies immediately following the Daya Bay results showed that the physics reach for muon energies in the range $10 \mathrm{GeV}$ to $12.6 \mathrm{GeV}$ is essentially unchanged. Therefore a pragmatic approach was adopted as a full reoptimization of the accelerator chain in the available time was unrealistic. Work on the accelerator design had matured such that an initial costing based on the twoRLA option shown in Fig. 2 was available.

On the other hand, using a FFAG for the final stage of acceleration could offer cost benefits for energies up to $10 \mathrm{GeV}$ and the design of a $25 \mathrm{GeV}$ ring had been developed and costed [26,27]. As the redesign of the decay ring for $12.6 \mathrm{GeV}$ was faster to accomplish than to develop a new $10 \mathrm{GeV}$ design, the two RLA option delivering a final muon energy of $12.6 \mathrm{GeV}$ was adopted for the $\mathrm{EURO} \nu$ baseline neutrino factory and forms the basis of this report. After EURO $\nu$ finished and presented its final report, the design of a $10 \mathrm{GeV}$ FFAG was finalized. The one RLA and one FFAG option will be treated as an alternative to the baseline muon-acceleration scheme in this document.

\section{B. Proton driver}

At the start of the accelerator chain, a proton driver capable of delivering an average power of $4 \mathrm{MW}$ is required [28]. Several boundary conditions define the proton-beam parameters necessary to produce the desired number $\left(10^{21}\right.$ muon decays per year) of muons in the storage ring. The proton-beam energy must be in the multi-GeV range in order to maximize pion production [29]. In addition, the neutrino factory requires a particular time structure for injection into the decay ring consisting of three short bunch trains separated by $120 \mu \mathrm{s}$ [30]. The short bunch length of 1-3 ns rms is dictated by the efficiency of the muon-beam capture and the bunch separation is constrained by beam loading in the downstream muon accelerator and the recovery time of the mercury-jet target [31]. In order to achieve such short bunches, a dedicated bunch-compression scenario needs to be designed to deal with very strong space-charge forces. The main parameters of the proton driver are listed in Table I.

Several proton-driver schemes fulfilling these requirements have been proposed, and site-specific proton drivers for CERN, FNAL, and RAL have been presented in the 
TABLE I. Proton driver requirements. A proton kinetic energy in the range $5 \mathrm{GeV}$ to $15 \mathrm{GeV}$ has been shown to provide adequate performance [21]. The number of protons, beam radius, $\beta^{*}$, and geometric emittance correspond to the values for an $8 \mathrm{GeV}$ proton beam.

\begin{tabular}{lc}
\hline \hline Parameter & Value \\
\hline Kinetic energy & $5-15 \mathrm{GeV}$ \\
Average beam power & $4 \mathrm{MW}$ \\
& $\left(3.3 \times 10^{15} \mathrm{protons} / \mathrm{s}\right)$ \\
Repetition rate & $50 \mathrm{~Hz}$ \\
Bunches per train & 3 \\
Total time for bunches & $240 \mu \mathrm{s}$ \\
Bunch length (rms) & $1-3 \mathrm{~ns}$ \\
Beam radius & $1.2 \mathrm{~mm}(\mathrm{rms})$ \\
Rms geometric emittance & $<5 \mu \mathrm{m}$ \\
$\beta^{*}$ at target & $\geq 30 \mathrm{~cm}$ \\
\hline \hline
\end{tabular}

IDS-NF IDR [21]. The CERN solution is based upon the superconducting proton linac (SPL) [32,33] and employs a high power linac, followed by an accumulator and a compressor ring. In this scenario the beam time-structure is obtained with the help of charge-exchange injection into the accumulator ring, followed by fast phase-rotation in the compressor ring.

At RAL, the attractive idea of a common proton driver for the spallation-neutron source and the neutrino factory was proposed in the framework of the ongoing megawatt ISIS upgrade study $[34,35]$. In such a scenario the beam for both facilities will share the same source, chopper, linac, accumulation, and acceleration to $3.2 \mathrm{GeV}$ in a Rapid
Cycling Synchrotron (RCS) (see Fig. 3). After extraction, all bunches except for three will be sent directly to the neutron-spallation target, the three remaining will be injected into another RCS where, after acceleration and bunch compression [36], the beam will be extracted toward the Neutrino Factory pion-production target. Schematic layouts of the two, site-specific, proton-driver layouts are shown in figure 3 . Further details of the proton driver for a neutrino factory can be found in $[37,38]$.

\section{Target and pion capture}

The target station at the neutrino factory presents substantial engineering challenges. The target is required to produce and capture sufficient pions while simultaneously dissipating in excess of $1 \mathrm{MW}$ of proton beam power. The target must operate in the high magnetic field which maximizes the capture of large transverse momentum pions [42]. Extensive studies of the target have been performed to find the optimal proton-beam energy, the target material best suited to produce the required muon yield, the optimum magnetic-field distribution of the capture solenoid and the overall best target-station geometry $[43,44]$.

Pion production and the distribution of the dissipated power in the target area have been studied for solid and powder-jet targets of different materials and for liquid-mercury targets [29]. Yield calculations have been used to optimize the proton-driver energy $\left(E_{p}\right)$ resulting in the specification that $E_{p}$ should lie in the range $5 \mathrm{GeV}<E_{p}<10 \mathrm{GeV}$.
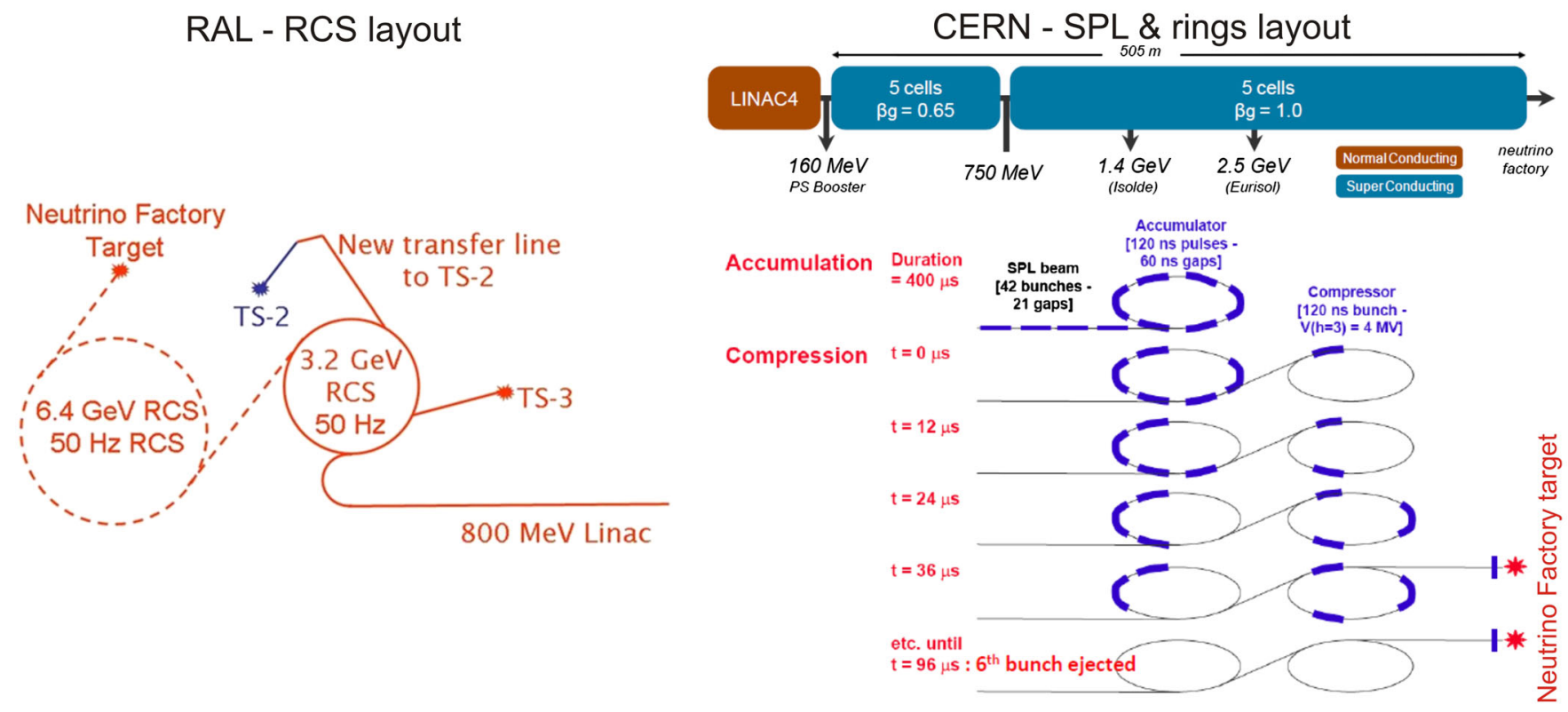

FIG. 3. The proposed $4 \mathrm{MW}$ proton drivers for a neutrino factory fall in two categories. The RAL option (left panel) consists of a medium energy linac injecting into two rapid-cycling synchrotrons delivering beam to the spallation-neutron targets and the neutrino factory target [35]. The CERN option (right panel) consists of the SPL linac [39,40] followed by an accumulator and compressor ring [41]. 

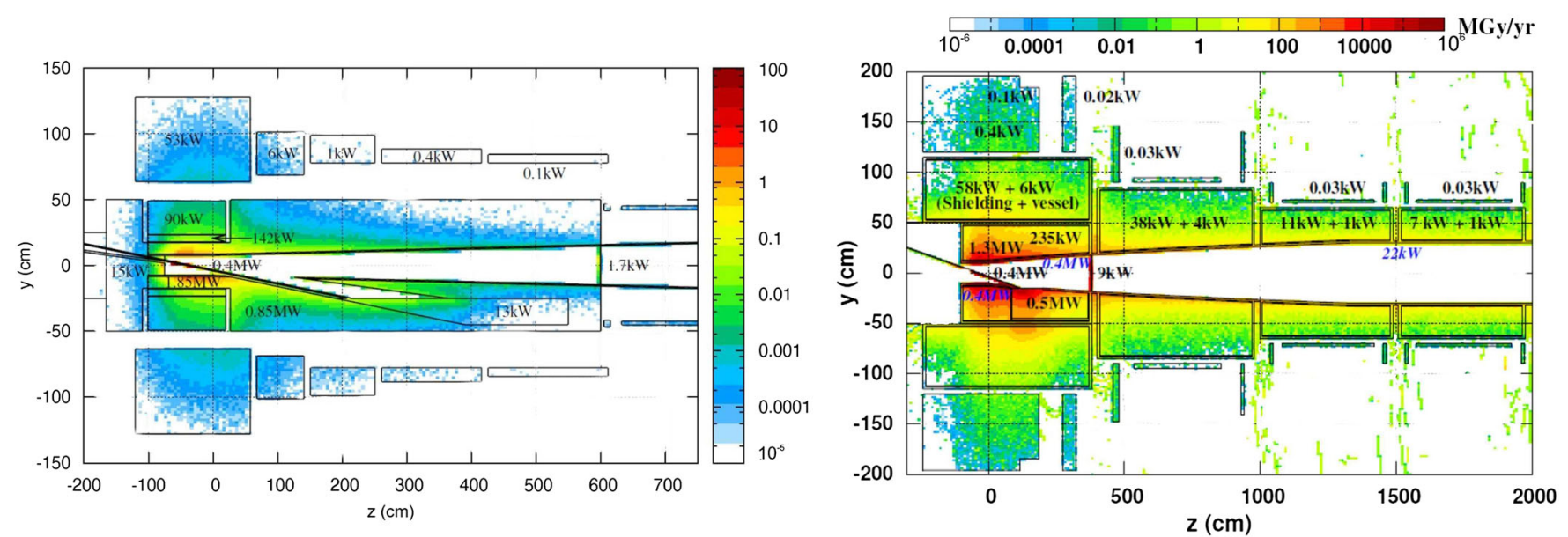

FIG. 4. Simulation of power dissipation due to radiation in the target station region for the Study 2a target design [56] (left panel) and the $\mathrm{EURO} \nu$ baseline target station (right panel).

A free flowing liquid-mercury-jet target has been chosen as the baseline for the neutrino factory. At the moment, this is the only technique that has been shown to be capable of operating in a multi-MW beam [45] and delivering the required muon yield [46]. The free-flowing jet has the advantage that the portion of the jet disrupted by the beam is replaced in time for the next proton-beam pulse. The measurements made by the MERIT collaboration [45] indicate that the mercury-jet technology is capable of operating successfully with the proton beam parameters of the neutrino factory. However, the liquid-mercury technology has the disadvantage that the mercury delivery and recirculation system is complex and the mercury itself presents substantial safety issues that must be overcome in the design of the target station. Therefore, solid and powder-jet target options have been investigated as a backup solution to mitigate the risks implied by the potential hazards of a liquid-mercury target [21].

The main concern regarding the use of a solid target was that the fatigue, induced by the shock introduced in the material by each successive proton bunch, would seriously limit the lifetime of the target. Simulations of the shock have been augmented with experimental studies in which the effect of the beam is simulated using a current pulse passing through various candidate materials [47]. Measurements using a Laser Doppler Vibrometer [48] have been compared with dynamic finite-element analysis using LS-DYNA [49]. The results obtained show that, while tantalum is too weak, tungsten has a sufficient lifetime at the neutrino factory as long as a mechanism to exchange the tungsten rods between beam pulses can be implemented [50]. Design work on a solid-target station, based on a rotating wheel bearing a number of tungsten rods has been initiated [51]. In view of the high level of the technical risk associated with the neutrino factory target, a prototype was constructed. A powder-jet target test rig has also been constructed and initial results from the powder-jet tests are promising [52].

For a proton energy of $10 \mathrm{GeV}$, a large fraction of the energy is dissipated near the target, with the consequence

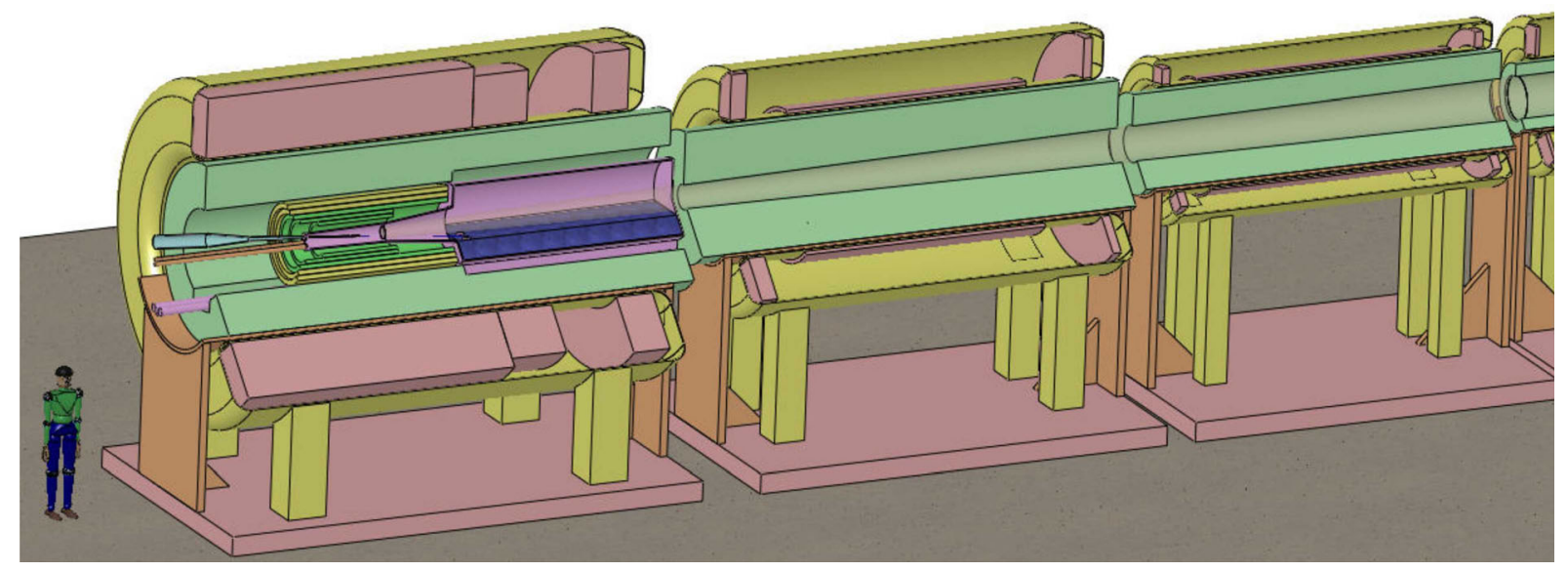

FIG. 5. Schematic of the EURO $\nu$ baseline Neutrino Factory target station using a free flowing mercury jet and capture solenoid. 
that effective shielding of the superconducting solenoids is required. Recent work showed [53] that, for the target station design presented in the IDR, the heat load expected in the superconducting coils would exceed technical limitations (see figure 4 left panel) and an improved layout was produced (see figure 4 right panel), reducing the heat load to acceptable levels $[53,54]$. Since then, the engineering design has progressed well and the physics design of the target station has been altered to accommodate the engineering constraints. A change in the design of the capture solenoids towards a modular design (see Fig. 5) reduced the maximum field strength in the interaction region from $\sim 20 \mathrm{~T}$ to $\sim 15 \mathrm{~T}$ and broadened the distribution of pions produced, increasing the pion yield by more than $10 \%$ [55]. Due to the increased shielding required for the superconducting capture solenoids, the requirements for shielding in the target building have been reduced, and in consequence the design of the Study 2a target station building [56] can be used with minor changes. A more detailed description can be found in [57].

\section{Muon front-end}

The muon front-end is designed to optimize the number of muons that can be transmitted through the downstream accelerator complex. Immediately after the target, the baseline design has a $15 \mathrm{~m}$ solenoid capture channel, with fields tapering from $15 \mathrm{~T}$ down to $1.5 \mathrm{~T}$, followed by a decay section of $\sim 58 \mathrm{~m}$. The muons then undergo adiabatic bunching in a $33 \mathrm{~m}$ long system of RF cavities of modest gradient ( $4 \mathrm{MV} / \mathrm{m}$ to $7.5 \mathrm{MV} / \mathrm{m}$ ) to produce a 201.25 MHz bunch structure. This is followed by an RF phase-rotation section $42 \mathrm{~m}$ in length with higher gradients $(12 \mathrm{MV} / \mathrm{m})$ and frequencies that decrease with distance down the channel. In this way, the energy spread is reduced and the beam is formed into bunch trains that are $\sim 80 \mathrm{~m}$ long. This process is shown schematically in the lower panel of Fig. 6. The phase-rotation section is followed by an ionization-cooling channel that is $\sim 75 \mathrm{~m}$ long in which the number of muons within the acceptance of the downstream muon accelerators is increased. The cooling section requires $201.25 \mathrm{MHz}$ cavities with high gradients $(15 \mathrm{MV} / \mathrm{m})$ in the presence of a high magnetic field. The cooling channel uses lithium hydride as an absorber material.

Since the publication of the IDS-NF IDR, two major changes have been included to produce the baseline muon front-end setup shown in the top panel of Fig. 6. A chicane [58] has been added between the pion decay channel and the lattice of the adiabatic buncher was adopted following engineering constraints. The chicane consists of a solenoidal transport channel with a beryllium absorber at its end. The chicane will remove unwanted secondary particles (mainly protons) from the beam to avoid activation downstream in the muon accelerator. Particle-dynamics studies show a good suppression of low- and high-momentum protons [58]. Future work will concentrate on the development of an engineering design for the chicane with emphasis on the particle absorbers.

Experiments at the Muon Test Area (MTA) [59] at Fermilab indicated that the strong magnetic fields required to transport the muon beam through the cooling channel may result in a significant reduction in the gradients that can be achieved in the cavities [60]. Since the experimental

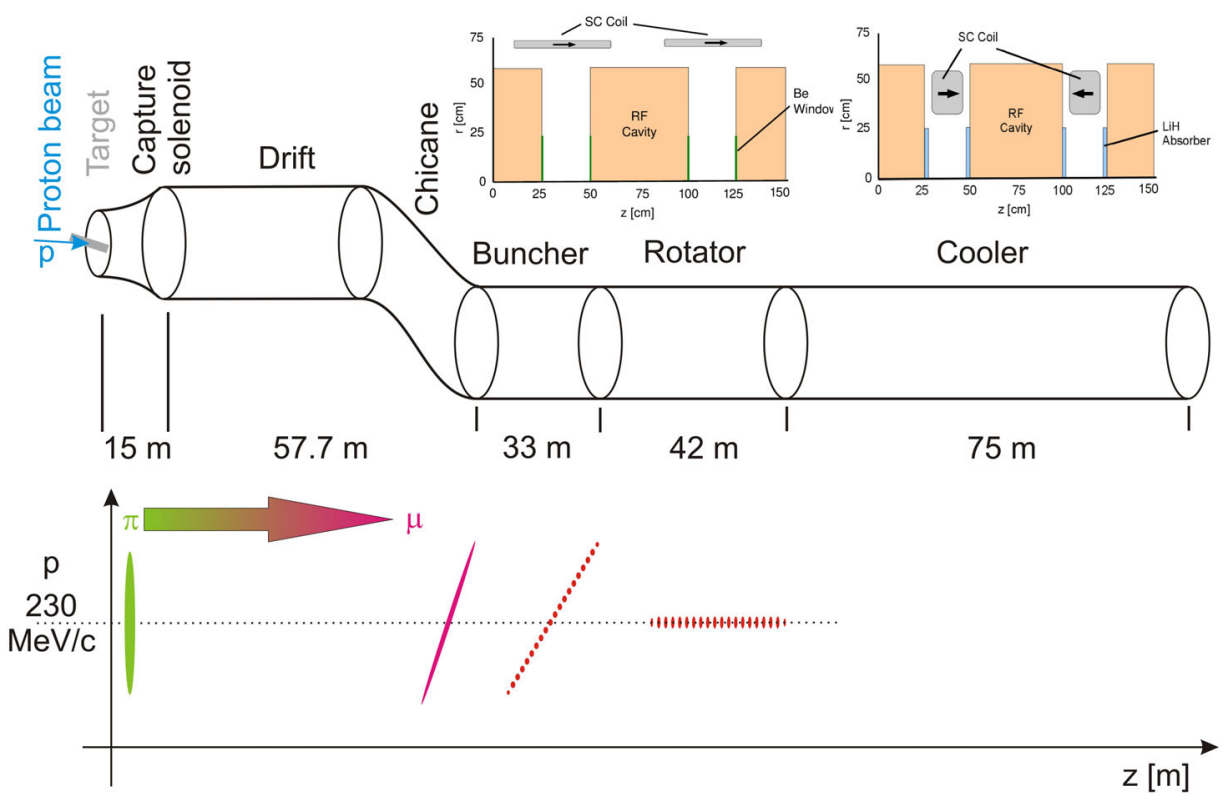

FIG. 6. Schematic of the neutrino factory front-end consisting of a chicane built with solenoids, a buncher and phase-rotation section to adjust the longitudinal phase space to the requirements of the following accelerator, and a cooling section to reduce the transverse emittance to allow for cost-effective acceleration. 


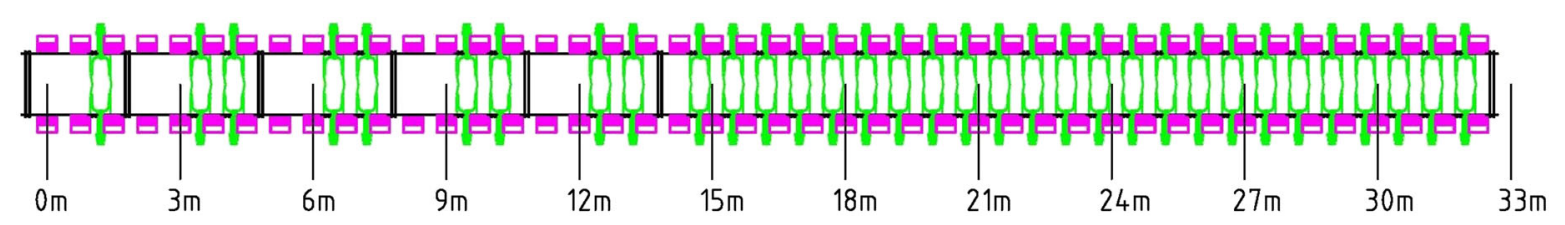

FIG. 7. Schematic of the bunching section. The lattice layout was adjusted to take into account several engineering constraints (RF feed-through, etc.) and to allow for a modular setup.

work is still in progress, the limiting gradient is presently unknown. To mitigate the risk that the performance of the channel will be compromised, various alternative lattices have been studied and the use of pressurized gas-filled $\mathrm{rf}$ cavities and cavity-surface treatments have been considered [61]. A number of the alternative lattices have been shown to perform at a level that is competitive to the baseline at a significantly reduced risk of breakdown. The most promising candidate is the "bucked coil" lattice [62-65].

Engineering considerations have resulted in some modifications to the bunching lattice presented in the IDR [21] (see Fig. 7). The impact of these changes on the performance of the channel will be studied and further design changes to mitigate any resulting performance reductions will be considered. Future studies within the IDS-NF will focus on the implementation of alternative cooling-lattice options, in particular a bucked-coil configuration and a lattice in which the solenoids have additional iron shielding that reduces the magnetic field at the cavities. Further details can be found in [66].

\section{E. Muon acceleration}

The baseline $\mathrm{EURO} \nu$ neutrino factory muon-accelerator complex will accelerate muons to $12.6 \mathrm{GeV}$. As was explained earlier, two options for the muon accelerator chain are being considered. The first (scheme 1) exploits a linac followed by two recirculating linear accelerators (RLAs) while in the second (scheme 2) a nonscaling FFAG replaces the second RLA. EURO $\nu$ has adopted scheme 1 as the baseline. In line with developments within the IDS-NF collaboration, scheme 2, with a final muon energy of $10 \mathrm{GeV}$, is maintained as an alternative.

Acceleration of the muon beams will be achieved in three stages (see Fig. 8) [67]. The chain of accelerators is optimized by maximizing the efficiency at each stage.
Two muon-acceleration schemes are under investigation within the IDS-NF. A solenoid-focused linac first accelerates the beam to a total energy of either $0.9 \mathrm{GeV}$ (scheme 1) or $1.2 \mathrm{GeV}$ (scheme 2). Then, a dog-bone recirculating linear accelerator with FODO cells accelerates the beam to $3.6 \mathrm{GeV}$ (scheme 1) or $5 \mathrm{GeV}$ (scheme 2). The final acceleration is performed using either a second RLA, accelerating from $3.6 \mathrm{GeV}$ to $12.6 \mathrm{GeV}$ in 4.5 passes (scheme 1), or a fixed field alternating gradient (FFAG) accelerator (scheme 2), accelerating from $5 \mathrm{GeV}$ to $10 \mathrm{GeV}$ in $\sim 9$ turns. Since the IDR was finalized, the linac and RLA lattices have been revised to improve the transverse acceptance and simplify the design of the switch yards. All accelerating cavities are $201.25 \mathrm{MHz}$ superconducting structures.

The muon accelerators require a transverse phase-space acceptance of $30 \pi \mathrm{mm} \mathrm{rad}$. Such an acceptance is enormous compared with conventional beams in proton and electron machines. The muon life-time at rest is $2.2 \mu \mathrm{s}$, so acceleration has to be rapid to benefit from the effects of time dilation and to reduce decay losses. While FFAG accelerators are promising in this respect they work less efficiently at low energies. Therefore the baseline system starts with a $201.25 \mathrm{MHz}$ linac which accelerates the beam to $0.9 \mathrm{GeV}$ while adiabatically reducing the phase-space volume. The design has been simulated using the OptiM [68] and MAD-X [69] codes and the mechanical design and electromagnetic simulations of the linac components (solenoids and cavities) have been performed (see Fig. 9) [70-72].

The design of the niobium-sputtered superconducting cavities is straightforward. The solenoid design is complicated by the need to reduce the magnetic fringe field at the position of the cavities (to avoid reducing the achievable accelerating gradient) using a counterexcited

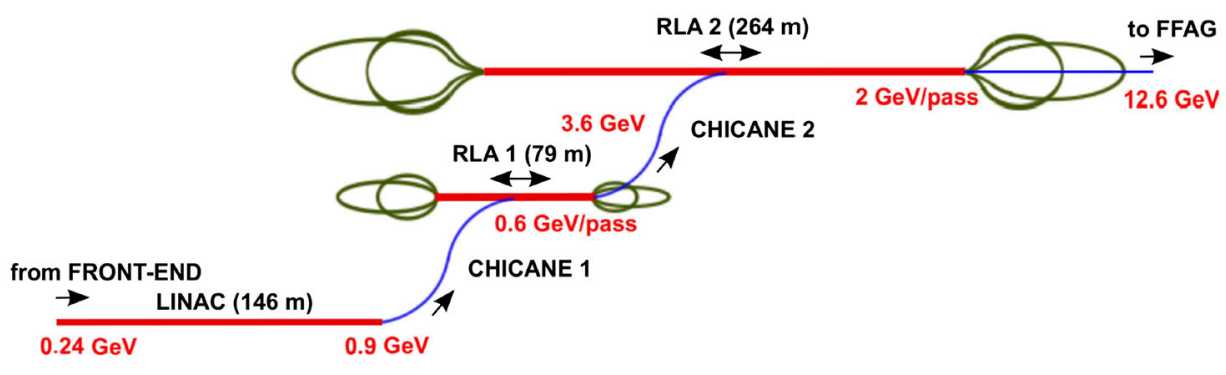

FIG. 8. Schematic layout of the muon acceleration to $12.6 \mathrm{GeV}$ for the baseline lattice (scheme 1) showing the status in April 2012. 


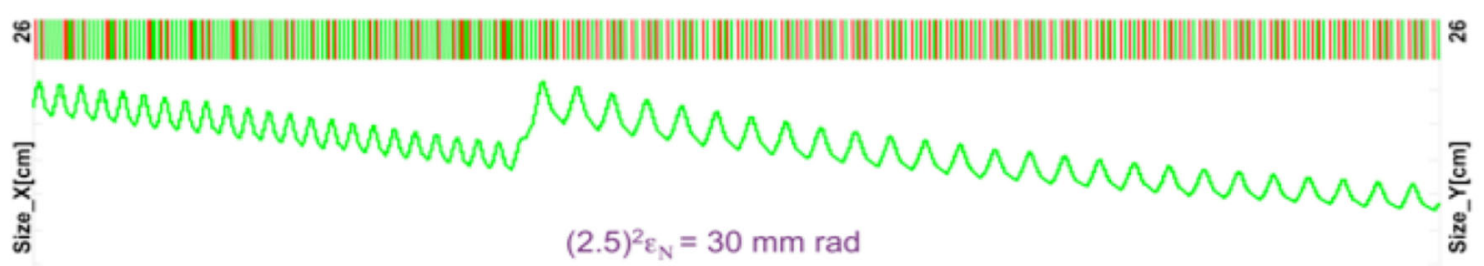

$(2.5)^{2} \sigma_{\Delta p} \sigma_{z} / m_{\mu} c=150 \mathrm{~mm}$
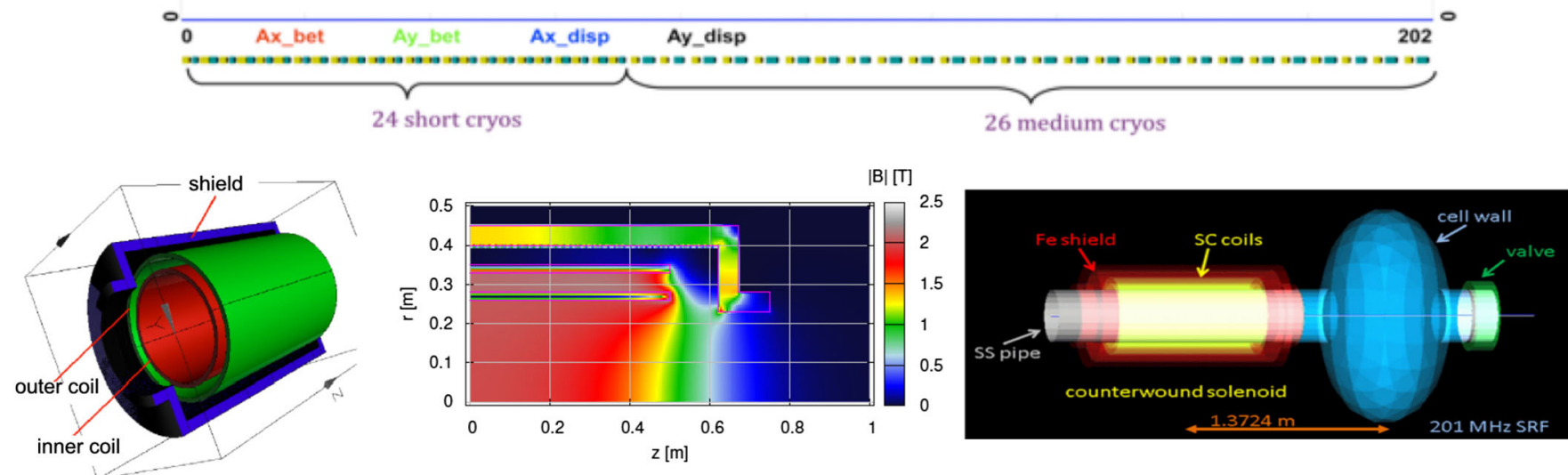

FIG. 9. Top panel: Layout of the muon linac consisting of 24 one cavity modules and 26 two cavity modules to achieve a final energy of $0.9 \mathrm{GeV}$. The lower left-hand panel shows an electromagnetic model of the superconducting solenoid, the lower center panel shows the field distribution at the end of the solenoid and the lower right-hand panel shows one short cryo-module.

outer shell (bucked coils) together with an iron shield. Multiparticle simulations, based on the field maps obtained from the results of the electromagnetic simulations, have been performed using GPT [73] and other codes. The huge momentum spread within a bunch causes particle losses in the first part of the linac to be $\sim 5 \%$ if a matching section between the cooling section and the linac is assumed. At the end of the linac, the beam has an energy of $0.9 \mathrm{GeV}$ (scheme 1) or $1.2 \mathrm{GeV}$ (scheme 2). The increased energy in scheme 2 is achieved by adding two cryo modules to the end of the scheme-1 linac. The energy at the end of the linac allows a recirculating linear accelerator to be used as the phase slip (caused by the variation in time-of-flight with energy) is tolerable. Dog-bone RLAs give improved cost efficiency over normal linacs and racetrack RLAs, but features such as the nonzero energy spread in the beam, the transverse beam size and the space required for magnet coils restrict the number of separate return arcs into which the beam can be directed and so limit the number of passes through the accelerating structures.

After the linac, the beam is injected into a dog-bone RLA via the injection chicane shown in figure 10 . The beam makes 4.5 passes in RLA1, gaining $0.6 \mathrm{GeV}$ of energy per pass for scheme 1 or $\sim 0.84 \mathrm{GeV}$ energy per pass for scheme 2. An example of the arc design is shown in Fig. 11. In the case of the two-RLA scenario, a second RLA

20
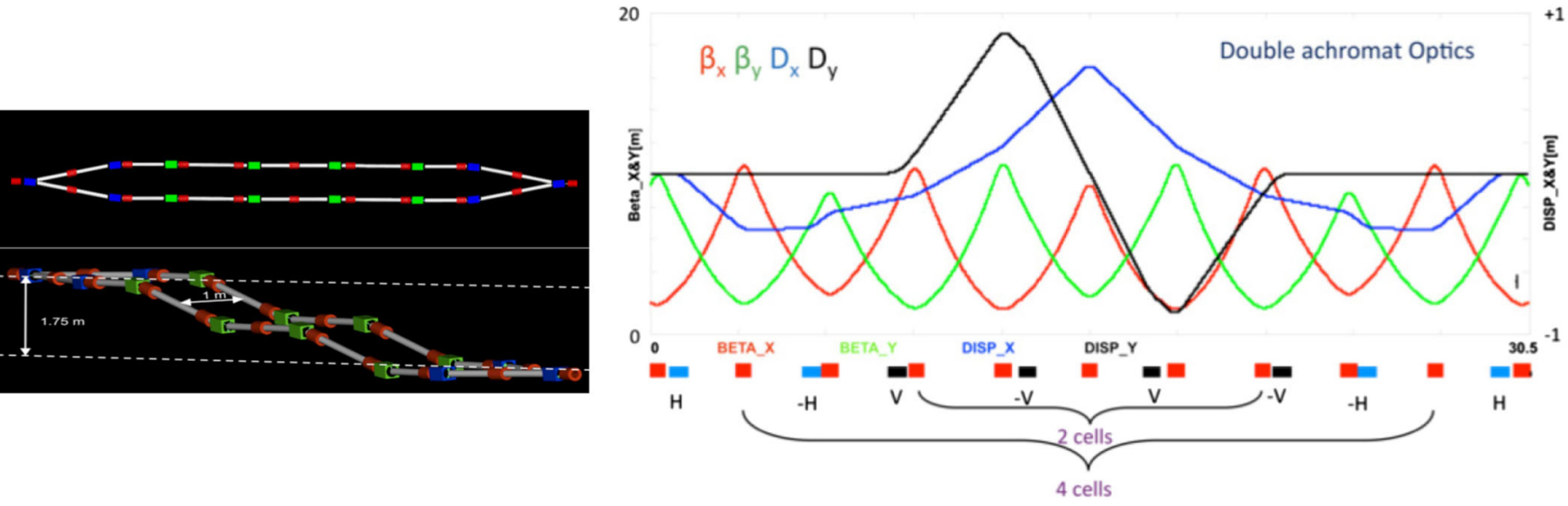

FIG. 10. Left: Layout of the injection chicane from the muon linac into the first RLA in two projections. Right: lattice details of the injection chicane in OptiM. 


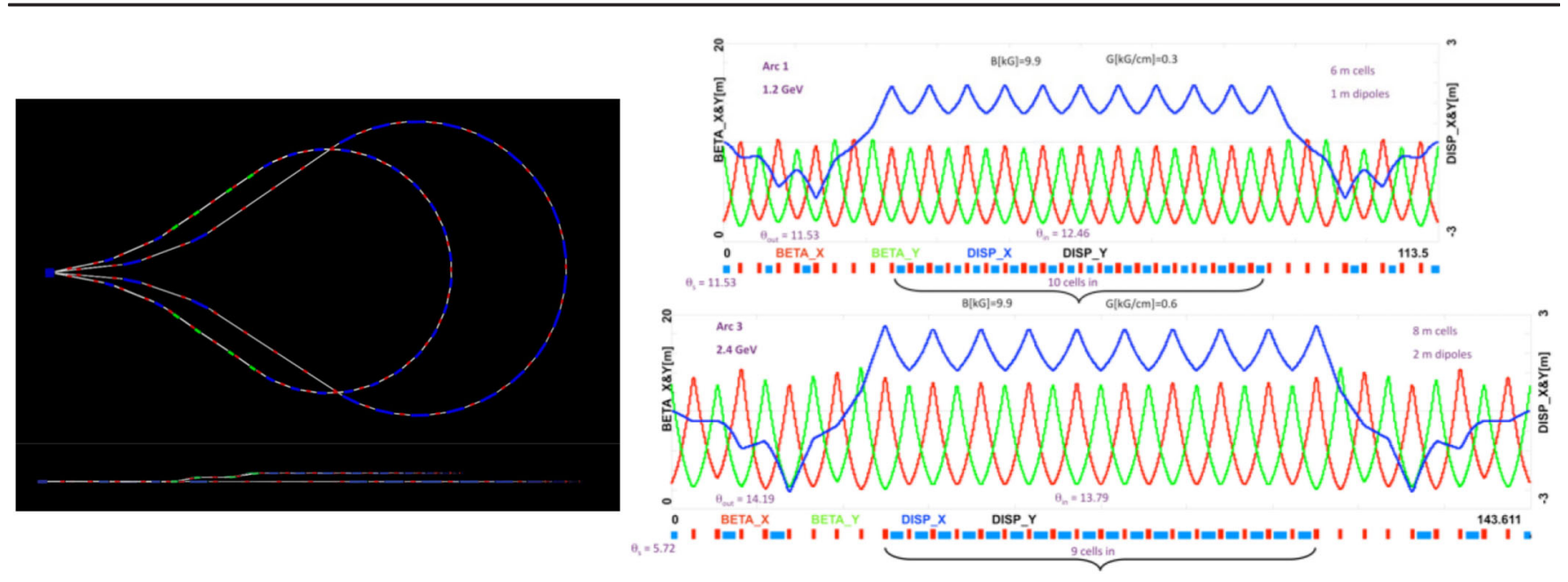

FIG. 11. Left: Layout of the recirculation arcs for $1.2 \mathrm{GeV}$ and $2.4 \mathrm{GeV}$ of the first RLA in two projections. Right: lattice details of the arcs in OptiM.
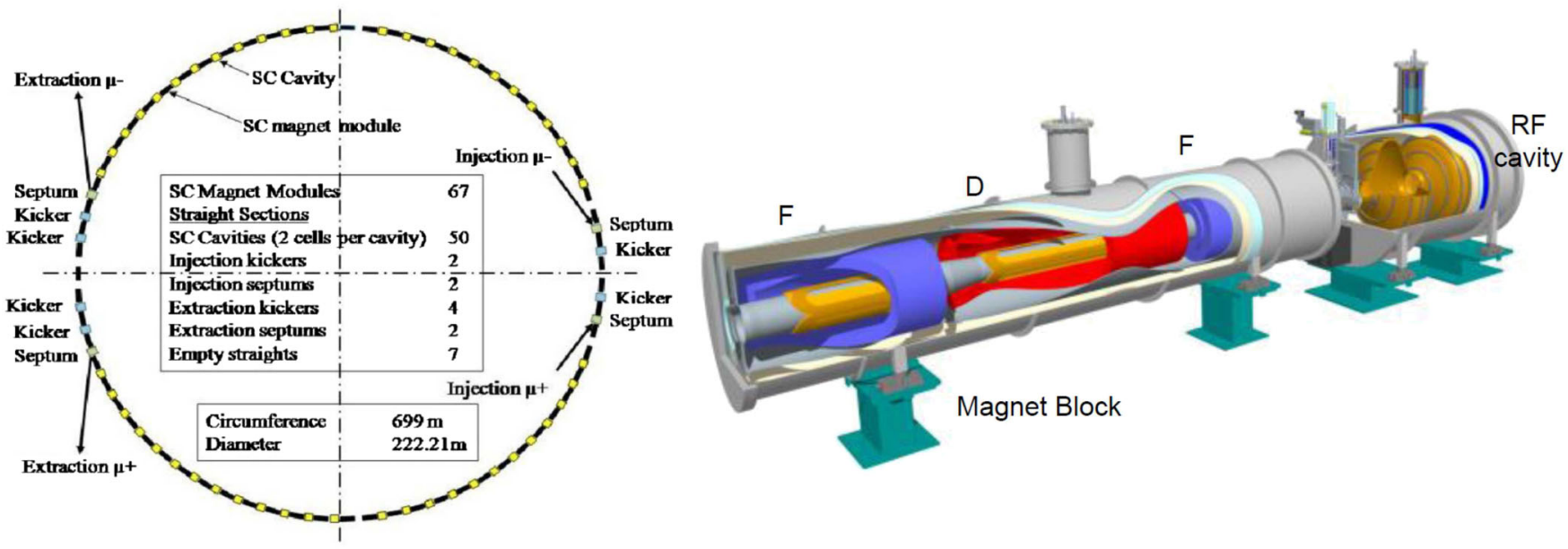

FIG. 12. Left: NS-FFAG layout presented in the IDS-NF IDR [21]. Right: engineering concept for the cryo-modules including three magnets and one cavity.

accelerates the beam from $3.6 \mathrm{GeV}$ to $12.6 \mathrm{GeV}$ again in 4.5 passes. Further details can be found in [74].

The second scenario under investigation uses a nonscaling FFAG (NS-FFAG) accelerator of similar design to that originally proposed for the final muon acceleration in the IDS-NF IDR [26,75]. It is a quasi-isochronous design (using serpentine acceleration) and allows for up to 9 turns in the lattice filled with $201.25 \mathrm{MHz}$ RF cavities, increasing the muon acceleration efficiency. The advantage of a linear, strong-focusing NS-FFAG lattice is that it can transport a large emittance muon beam, using relatively compact magnets since the orbit excursion is small. A full design of this lattice, including an injection and extraction scheme, was presented in the IDR [21]. Over the past year, initial engineering studies (see figure 12) have been carried out [76]. The principle of this novel type of accelerator has been demonstrated in the EMMA proof-of-principle machine [77].
One remaining difficulty is the variation in time-of-flight for particles with large transverse amplitude. This causes phase slip at the RF cavities which can lead to distortion of the longitudinal phase-space and an increase in the energy spread at extraction. Despite the fact that this increase in energy spread is much larger than the effect of beam

TABLE II. Final acceleration using the NS-FFAG. Comparison of design parameters for the fully designed and costed 12.625 GeV FFAG [21] with the first results for the 5-10 GeV FFAG proposed as an alternative to the two-RLA solution.

\begin{tabular}{lcc}
\hline \hline Parameter & 25 GeV FFAG & $10 \mathrm{GeV}$ FFAG \\
\hline Circumference [m] & 669 & 328.8 \\
RF voltage MV & 1196 & 550 \\
Number of cells & 67 & 49 \\
magnetised length [m] & 263 & 108.3 \\
\hline \hline
\end{tabular}




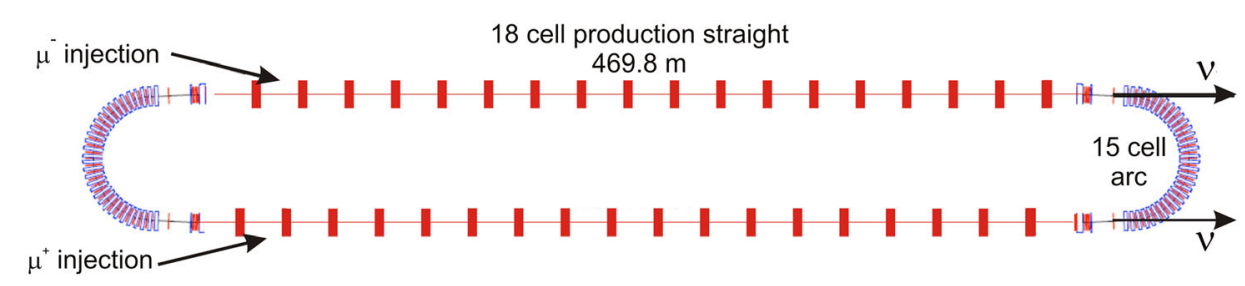

FIG. 13. Schematic of the racetrack muon decay ring.

loading [78], the resulting beam can still be accepted by the decay ring. It has been shown that chromaticity correction, performed by introducing nonlinear magnetic-field components, can diminish the longitudinal distortion and the final energy spread [79]. The disadvantage of this solution is the reduction of the dynamic acceptance. The final level of chromaticity correction adopted will be a compromise between the reduction of phase-space distortion and the maintenance of a large acceptance. The important issues of injection and extraction have been addressed in recent studies $[27,80]$. Several lattice candidates were considered carefully with the result that a triplet solution with $3 \mathrm{~m}$ long straight sections is preferred. This choice is based on a compromise between the beam-dynamics considerations and the field requirements for the kicker and septum magnets. In the proposed injection/extraction geometries, several kicker units are distributed in several lattice cells, assuming mirror symmetry in order to reuse the same kickers for both signs of muon. In this way a sufficient orbit separation can be created allowing septum magnets to be used with strengths that require superconducting technology. The ring design presented in the IDS-NF IDR has been modified to accelerate from $5 \mathrm{GeV}$ to $10 \mathrm{GeV}$. First results are available with the main FFAG parameters shown in Table II. This first layout will allow for a better cost comparison [81] with the other scenario under discussion. Further details can be found in [74].

\section{F. Muon storage ring}

The neutrino factory design specifies one racetrack shaped storage ring pointed toward a detector sited at a distance of between $\sim 1500 \mathrm{~km}$ and $\sim 2500 \mathrm{~km}$ from the source [82]. The design of the $12.6 \mathrm{GeV}$ storage ring has been developed from the $25 \mathrm{GeV}$ ring that was included in the IDS-NF IDR [21,83], a $10 \mathrm{GeV}$ decay ring design was finalized recently [84]. This design doubles the neutrino rate to one detector by storing counterrotating positive and negative muon beams (see Fig. 13). The choice of geometry for the decay ring depends on the ratio of the total length of the neutrino-production straights to the circumference and
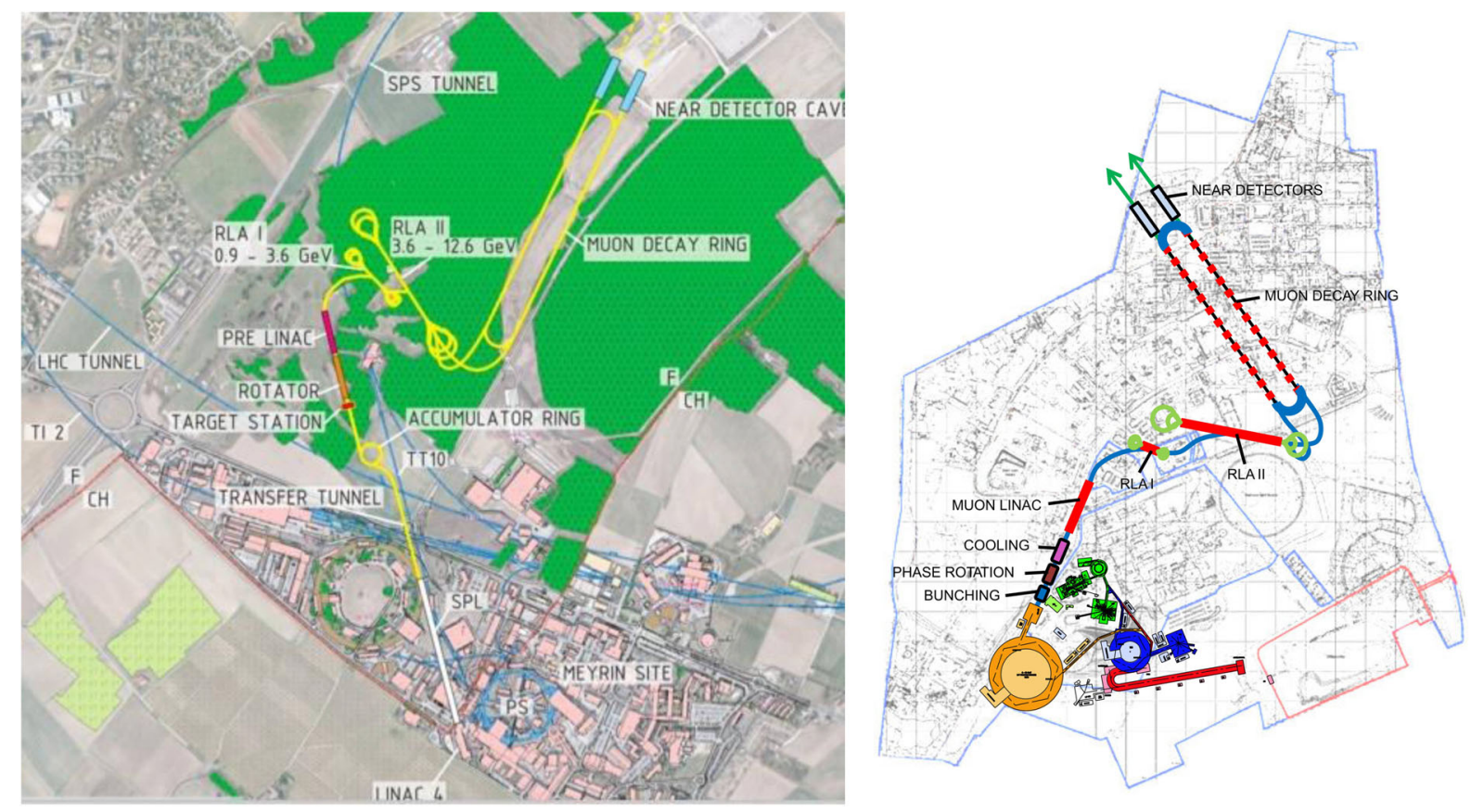

FIG. 14. Layout of the EURO $\nu$ baseline neutrino factory on the two sites considered by EURO $\nu$. Left: Layout at CERN in which SPL servers accumulator and compressor rings and the muon-accelerator complex is laid out in the North Area. Right: Layout at RAL in which an upgrade of ISIS serves an RCS and the muon-accelerator complex is laid out on the Harwell Campus. 
the depth of the tunnels, which has cost implications. The proposed decay ring is $1300 \mathrm{~m}$ in circumference and accommodates the equally-spaced, $250 \mathrm{~ns}$ long, bunch trains with time intervals of at least $120 \mu$ s between the neutrino bursts. The production straights for the race-track design are $\sim 470 \mathrm{~m}$ long, giving an efficiency per sign of stored muon of $36.15 \%$. The tunnel depth for a ring of this size is around $100 \mathrm{~m}$. To keep the neutrino beams reasonably well focused, the muon-beam rms divergence should not add more than $\sim 10 \%$ to the natural width of the decay cone. This means that the $\beta$ function, which should be small $(\sim 10 \mathrm{~m})$ in the arcs, has to be matched to values of about $80 \mathrm{~m}$ at the start of the long production straights which can be achieved by matching sections at the end of each straight. Simulations of the racetrack decay ring have been carried out using the code Zgoubi [85]. Additional simulations showed that, given the predicted energy spread of the muon beams, the bunches in the bunch train will not merge (bunch separation larger $100 \mathrm{~ns}$ ) before twice the lifetime of the muons and therefore no RF has to be installed in the decay ring. In the future, tracking studies will address the machine's dynamic aperture including errors. Additionally an efficient collimation system needs to be developed to cope with the muon beam power that the rings have to sustain. More information concerning the decay rings can be found in [84].

For the flux at the detector to be predicted it is essential that the parameters of the stored-muon beam are measured and studies of the beam instrumentation in the decay ring (beam current and beam divergence) have been performed. A near detector will be used to study neutrino-nucleus interactions and to allow the extrapolation of the neutrino flux to the position of the far detector [86].

\section{CONCLUSIONS}

The EURO $\nu$ Design Study consortium, in the context of the International Design Study for the neutrino factory (the IDS-NF) collaboration, has developed a self-consistent design for the facility. EURO $\nu$ has made substantial contributions to the significant progress that has been made in the accelerator physics and initial engineering studies for a neutrino factory. Two site-specific layouts have been produced for the neutrino factory at CERN and at RAL based on different proton-driver technologies (see Fig. 14). Various options for target material and proton energy have been investigated in terms of pion yield. While solid targets and fluidized power targets have been investigated, a concept for the implementation of these target options is not as well developed as that of the free-flowing mercuryjet target. The introduction of shielding between the target and the superconducting solenoids for pion capture has reduced the heat load to an acceptable level. A chicane has been introduced between the decay channel and the buncher section to control the loss of secondary particles and to decrease the activation in the downstream accelerator systems. The bunching section has been adapted to accommodate engineering constraints and alternative cooling lattices with performance comparable to the baseline have been developed. The first part of the muon acceleration has been further refined while the final acceleration step continues to be studied within the IDS-NF collaboration. The design of the decay ring has been revised to store $12.6 \mathrm{GeV}$ muon beams. The IDS-NF continues to develop the concepts for the facility in the preparation of its Reference Design Report.

\section{ACKNOWLEDGMENTS}

The work reported here was supported by the European Community under the European Commission Framework Programme 7 Design Study: EURO $\nu$, Project Number 212372. We also thank the Science and Technologies Facilities Council (UK) for the support of the work performed. We gratefully acknowledge the work of many of our colleagues within the muon beams for particle physics community without their contributions the progress in the design of a Neutrino Factory would have been very limited. We are indebted to the IDS-NF collaboration which has provided context within which the work reported has been carried out.

[1] J. Beringer et al. (Particle Data Group), Phys. Rev. D 86, 010001 (2012).

[2] F. An et al., Phys. Rev. Lett. 108, 171803 (2012).

[3] F. An et al., Chin. Phys. C 37, 011001 (2013).

[4] J. Ahn et al., Phys. Rev. Lett. 108, 191802 (2012).

[5] Y. Abe et al., Phys. Rev. D 86, 052008 (2012).

[6] Y. Abe et al., Phys. Rev. Lett. 108, 131801 (2012).

[7] K. Abe et al., Phys. Rev. Lett. 107, 041801 (2011).

[8] K. Abe et al. (T2K Collaboration), arXiv:1304.0841.

[9] K. Abe et al. (T2K Collaboration), arXiv:1308.0465.

[10] B. Pontecorvo, Sov. Phys. JETP 6, 429 (1957).

[11] B. Pontecorvo, Sov. Phys. JETP 7, 172-173 (1958).

[12] Z. Maki, M. Nakagawa, and S. Sakata, Prog. Theor. Phys. 28, 5 (1962).

[13] S. M. Bilenky, S. Pascoli, and S. T. Petcov, Phys. Rev. D 64, 053010 (2001).

[14] M. Gonzalez-Garcia, M. Maltoni, J. Salvado, and T. Schwetz, J. High Energy Phys. 12 (2012) 123.

[15] S. Geer, Phys. Rev. D 57, 6989 (1998).

[16] P. Coloma, A. Donini, E. Fernandez-Martinez, and P. Hernandez, J. High Energy Phys. 06 (2012) 073.

[17] A. Rubbia et al., "Expression of Interest for a very long baseline neutrino oscillation experiment (LBNO)." https:// cdsweb.cern.ch/record/1457543/files/SPSC-EOI-007.pd, 2012.

[18] M. Aoki, K. Hagiwara, and N. Okamura, Phys. Lett. B 554, 121 (2003).

[19] P. Zucchelli, Phys. Lett. B 532, 166 (2002).

[20] A. Bandyopadhyay et al., Rep. Prog. Phys. 72, 106201 (2009). 
[21] S. Choubey et al. (IDS-NF Collaboration), arXiv: 1112.2853.

[22] A. P. Blondel and J.-S. Graulich, in Proceedings of Cool07, Bad Kreuznach, Germany (Bad Kreuznach, Germany, 2007), p. TUM2I05.

[23] ISS Collaboration, "International scoping study of a future Neutrino Factory and super-beam facility." http://www.hep .ph.ic.ac.uk/iss/.

[24] S. Geer, O. Mena, and S. Pascoli, Phys. Rev. D 75, 093001 (2007).

[25] IDS-NF Collaboration, "The International Design Study for the Neutrino Factory." https://www.ids-nf.org/wiki/ FrontPage.

[26] J. S. Berg, M. Aslaninejad, J. Pasternak, H. Witte, N. Bliss et al., in Proceedings of the 2nd International Particle Accelerator Conference, San Sebastián Spain (EPS-AG, Spain, 2011), p. MOPZ007.

[27] J. Pasternak et al., in Proceedings of the 23rd Particle Accelerator Conference, Vancouver, Canada, 2009 (IEEE, Piscataway, NJ, 2009), p. WE6PFP092.

[28] H. G. Kirk et al., in Proceedings of the 10th European Particle Accelerator Conference, Edinburgh, Scotland, 2006 (EPS-AG, Edinburgh, Scotland, 2006), p. MOPCH138.

[29] J. Back et al., in Proceedings of the 2nd International Particle Accelerator Conference, San Sebastián, Spain (EPS-AG, Spain, 2011), p. MOPZ008.

[30] D. Kelliher et al., in Proceedings of the 3rd International Particle Accelerator Conference, New Orleans, LA, 2012 (IEEE, Piscataway, NJ, 2012), p. MOPPC030.

[31] K. McDonald et al., in Proceedings of the International Particle Accelerator Conference, Kyoto, Japan (ICR, Kyoto, 2010), p. WEPE078.

[32] R. Garoby et al., Report No. CERN-NEUTRINOFACTORY-NOTE-157, 2009.

[33] B. Autin et al., Report No. CERN-2000-012.

[34] D. J.S. Findlay, in Proceedings of the 22nd Particle Accelerator Conference, PAC-2007, Albuquerque, NM (IEEE, New York, 2007), p. TUYKI01.

[35] J. Pasternak and J. Thomason, in Proceedings of the 2nd International Particle Accelerator Conference, San Sebastián Spain (EPS-AG, Spain, 2011), p. WEPS105.

[36] L. Jenner and J. Pasternak, in Proceedings of the 2nd International Particle Accelerator Conference, San Sebastián Spain (EPS-AG, Spain, 2011), p. WEPS103.

[37] J. W. G. Thomason, R. Garoby, S. Gilardoni, L. J. Jenner, and J. Pasternak, Phys. Rev. ST Accel. Beams 16, 054801 (2013).

[38] J. Pasternak et al., in Proceedings of the 4th International Particle Accelerator Conference, IPAC-2013, Shanghai, China, 2013 (JACoW, Shanghai, China, 2013), p. TUPFI074.

[39] F. Gerigk et al., CERN Technical Report No. CERN-2006006, 2006.

[40] R. Garoby et al., in Proceedings of the 2nd International Particle Accelerator Conference, San Sebastián Spain (EPS-AG, Spain, 2011), p. WEPS017.

[41] E. Benedetto, in Proceedings of the 11th International Workshop on Neutrino Factories, Superbeams and Beta
Beams, July 20-25, 2009 (Illinois Institute of Technology, Chicago, 2009).

[42] J. Back, H. Kirk, N. Souchlas et al., in Proceedings of the 2nd International Particle Accelerator Conference, San Sebastián Spain (EPS-AG, Spain, 2011), p. MOPZ008.

[43] S. Brooks et al., in Proceedings of the 10th European Particle Accelerator Conference, Edinburgh, Scotland, 2006 (EPS-AG, Edinburgh, Scotland, 2006), p. WEPLS001.

[44] S. Brooks, in Proceedings of the International Particle Accelerator Conference, Kyoto, Japan (ICR, Kyoto, 2010), p. WEPE098.

[45] H. G. Kirk et al., in Proceedings of the 11th European Particle Accelerator Conference, Genoa, 2008 (EPS-AG, Genoa, Italy, 2008), p. WEPP169.

[46] I. Efthymiopoulos et al., in Proceedings of the 11th European Particle Accelerator Conference, Genoa, 2008 (EPS-AG, Genoa, Italy, 2008), p. MOPC087.

[47] G. P. Skoro et al., in Proceedings of the 23rd Particle Accelerator Conference, Vancouver, Canada, 2009 (IEEE, Piscataway, NJ, 2009), p. WE6RFP039.

[48] http://www.metrolaserldv.com/.

[49] http://www.ls-dyna.com/.

[50] G. P. Skoro et al., in Proceedings of the International Particle Accelerator Conference, Kyoto, Japan (ICR, Kyoto, 2010), p. THPEC091.

[51] T. Edgecock et al., in Proceedings of the International Particle Accelerator Conference, Kyoto, Japan (ICR, Kyoto, 2010), p. THPEC089.

[52] O. Caretta et al., in Proceedings of the 11th European Particle Accelerator Conference, Genoa, 2008 (EPS-AG, Genoa, Italy, 2008), p. WEPP161.

[53] N. Souchlas, R. Weggel, H. Kirk et al., in Proceedings of the 2nd International Particle Accelerator Conference, San Sebastián Spain (EPS-AG, Spain, 2011), p. TUPS054.

[54] N. Souchlas et al., in Proceedings of the 24th Particle Accelerator Conference, PAC-2011, New York, 2011 (IEEE, New York, 2011), p. TUP179.

[55] H. G. Kirk et al., in Proceedings of the International Particle Accelerator Conference, Kyoto, Japan (ICR, Kyoto, 2010), p. WEPE101.

[56] C.H. Albright et al. (Neutrino Factory/Muon Collider Collaboration), arXiv:physics/0411123.

[57] J. J. Back, C. Densham, R. Edgecock, and G. Prior, Phys. Rev. ST Accel. Beams 16, 021001 (2013).

[58] C. Rogers, D. Neuffer, and P. Snopok, in Proceedings of the 3rd International Particle Accelerator Conference, New Orleans, LA, 2012 (IEEE, Piscataway, NJ, 2012), p. MOPPC041.

[59] M. Popovic, in Proceedings of the 9th European Particle Accelerator Conference, Lucerne, 2004 (EPS-AG, Lucerne, 2004), p. MOPLT118 [http://accelconf.web .cern.ch/AccelConf/e04/].

[60] R. Palmer et al., arXiv:0809.1633v1.

[61] D. Neuffer, A. Alekou, C. Rogers, P. Snopok, and C. Yoshikawa, "IDR Neutrino Factory Front End and Variations" (to be published).

[62] A. Alekou et al., in Proceedings of the International Particle Accelerator Conference, Kyoto, Japan (ICR, Kyoto, 2010), p. WEPE050. 
[63] A. Alekou, J. Pasternak, and C. Rogers, J. Phys. Conf. Ser. 408,012056 (2013).

[64] A. Alekou et al., in Proceedings of the 3rd International Particle Accelerator Conference, New Orleans, LA, 2012 (IEEE, Piscataway, NJ, 2012), p. TUPPD003.

[65] D. Stratakis et al., in Proceedings of the 4th International Particle Accelerator Conference, IPAC-2013, Shanghai, China, 2013 (JACoW, Shanghai, China, 2013), p. TUPFI087.

[66] D. Neuffer, G. Prior, C. Rogers, P. Snopok, and C. Yoshikawa, AIP Conf. Proc. 1382, 196 (2011).

[67] S. A. Bogacz, Nucl. Phys. B, Proc. Suppl. 155, 334 (2006).

[68] V. Lebedev, "OptiM." http://www-bdnew.fnal.gov/pbar/ organizationalchart/lebedev/OptiM/optim.htm.

[69] http://mad.web.cern.ch/mad/.

[70] M. Aslaninejad, C. Bonţoiu, J. Pasternak, J. Pozimski, and S. Bogacz, in Proceedings of the International Particle Accelerator Conference, Kyoto, Japan (ICR, Kyoto, 2010), p. THPEB035.

[71] C. Bonţoiu, M. Aslaninejad, J. Pozimski, and A. Bogacz, in Proceedings of the International Particle Accelerator Conference, Kyoto, Japan (ICR, Kyoto, 2010), p. THPEB033.

[72] A. Kurup, C. Bonţoiu, M. Aslaninejad, J. Pozimski., A. Bogacz et al., in Proceedings of the 2nd International Particle Accelerator Conference, San Sebastián Spain (EPS-AG, Spain, 2011), MOPZ009.

[73] S. B. van der Geer and M. J. de Loos, "General Particle Tracer," http://www.pulsar.nl/gpt/.

[74] S. Bogacz, V. Morozov, Y. Roblin, K. Beard, and A. Kurup, in Proceedings of the 3rd International Particle Accelerator Conference, New Orleans, LA, 2012 (IEEE, Piscataway, NJ, 2012), p. TUPPD008.

[75] J. Berg, H. Witte, M. Aslaninejad et al., in Proceedings of the 3rd International Particle Accelerator Conference,
New Orleans, LA, 2012 (IEEE, Piscataway, NJ, 2012), p. MOPPC049.

[76] J. Pasternak, M. Aslaninejad, and C. Bonţoiu, in Proceedings of the 2nd International Particle Accelerator Conference, San Sebastián Spain (EPS-AG, Spain, 2011), MOPZ006.

[77] S. Machida et al., Nat. Phys. 8, 243 (2012).

[78] J. Pozimski, M. Aslaninejad, C. Bonţoiu et al., in Proceedings of the International Particle Accelerator Conference, Kyoto, Japan (ICR, Kyoto, 2010), p. WEPE060.

[79] J. Berg et al., in Proceedings of the 3rd International Particle Accelerator Conference, New Orleans, LA, 2012 (IEEE, Piscataway, NJ, 2012), p. MOPPC049.

[80] H. Witte et al., in Proceedings of the 3rd International Particle Accelerator Conference, New Orleans, LA, 2012 (IEEE, Piscataway, NJ, 2012), p. THPPD049.

[81] A. Kurup, N. Bliss, N. Collomb, and A. Grant, in Proceedings of the 3rd International Particle Accelerator Conference, New Orleans, LA, 2012 (IEEE, Piscataway, NJ, 2012), p. TUPPD004.

[82] A. Bross, R. Wands, R. Bayes, A. Laing, F. Soler et al., Phys. Rev. ST Accel. Beams 16, 081002 (2013).

[83] D. Kelliher, C. Prior, N. Bliss et al., in Proceedings of the 3rd International Particle Accelerator Conference, New Orleans, LA, 2012 (IEEE, Piscataway, NJ, 2012), p. MOPC030.

[84] D. Kelliher et al., in Proceedings of the 4th International Particle Accelerator Conference, IPAC-2013, Shanghai, China, 2013 (JACoW, Shanghai, China, 2013), p. TUPFI049.

[85] F. Meot and J.S. Berg, “ZGOUBI.” http://zgoubi .sourceforge.net/.

[86] M. Bogomilov, Y. Karadzhov, R. Matev, R. Tsenov, A. Laing, and F. J. P. Soler, Phys. Rev. ST Accel. Beams 16, 081001 (2013). 